\title{
Near-infrared absorbing semitransparent organic solar cells
}

\author{
Jan Meiss, Felix Holzmueller, Roland Gresser, ${ }^{\text {a) }}$ Karl Leo, and Moritz Riede ${ }^{\text {b) }}$ \\ Institut für Angewandte Photophysik, Technische Universität Dresden, 01062 Dresden, Germany
}

(Received 8 September 2011; accepted 26 October 2011; published online 11 November 2011)

\begin{abstract}
We present efficient, semitransparent small molecule organic solar cells. The devices employ an indium tin oxide-free top contact, consisting of thin metal films and an additional organic capping layer for enhanced light in/outcoupling. The solar cell encorporates a bulk heterojunction with the donor material $\mathrm{Ph}_{2}$-benz-bodipy, an infrared absorber. Combination of $\mathrm{Ph}_{2}$-benz-bodipy with $\mathrm{C}_{60}$ as acceptor leads to devices with high open circuit voltages of up to $0.81 \mathrm{~V}$ and short circuit current densities of 5-6 mA/cm $\mathrm{cm}^{2}$, resulting in efficiences of $2.2 \%-2.5 \%$. At the same time, the devices are highly transparent, with an average transmittance in the visible range (400-750 nm) of up to $47.9 \%$, with peaks at $538 \mathrm{~nm}$ of up to $64.2 \%$ and an average transmittance in the yellow-green range of up to 61.8\%. (C) 2011 American Institute of Physics. [doi:10.1063/1.3660708]
\end{abstract}

Organic solar cells are advancing towards commercialization, and materials and concepts have resulted in certified efficiencies $\eta$ of $8.3 \%$ for small molecule organic solar cells (SMOSCs) and for polymer devices. ${ }^{1}$ Building-integrated photovoltaics (BIPV) is expected to play an important role in the future. ${ }^{2}$ One field of BIPV includes the possibility of electricity-generating windows by fabricating semitransparent SMOSC, which would additionally provide sun shading. The development of optimized semitransparent metal contacts $^{3}$ and light incoupling layers ${ }^{4,5}$ finally led to $\eta$ approaching $5 \%$ in an optimized transparent tandem SMOSC with complementary absorbers; ${ }^{6}$ however, such devices had low transmittance $T$ in the visible spectrum. Recently, improved semitransparent devices ${ }^{7}$ with chloraluminium phthalocyanine $\mathrm{ClAlPc}$ as near-infrared (NIR) absorber achieved up to $\eta=1.7 \%$ with considerably higher $T$.

In this letter, we present a transparent SMOSC that employs benzannulated difluoro-bora-bis-(1-phenyl-indoyl)azamethine $\left(\mathrm{Ph}_{2}\right.$-benz-bodipy), described elsewhere, ${ }^{8}$ as nearinfrared absorbing donor material in combination with the acceptor $\mathrm{C}_{60}$. The material, shown in Fig. 1(a), has a highest occupied molecular orbital (HOMO) of $-5.22 \mathrm{eV}$ and a lowest unoccupied molecular orbital (LUMO) of $-3.65 \mathrm{eV}$ (determined by cyclovoltammetry $(\mathrm{CV})$ in dichloromethane ${ }^{8}$ ). $\mathrm{Ph}_{2}$-benz-bodipy has an absorption peak at $773 \mathrm{~nm}$, which makes this material extremely interesting for applications where transparency in the visible range combined with absorption in NIR is of importance.

Solar cells are fabricated in a custom-made vacuum system (K. J. Lesker, UK) at a base pressure of $10^{-8} \mathrm{mbar}$, using shadow masks, on $15 \times 15 \mathrm{~cm}^{2}$ glass wafers that contain a prestructured indium tin oxide (ITO) coating (Thin Film Devices Ltd, USA). The wafers are cleaned in a multistep wet process.

The complete solar cell stack is schematically shown in Fig. 1(b). The devices employ an inverted p-i-n-concept where highly conductive, doped charge transport layers sandwich the intrinsic absorber. An electron transport layer

\footnotetext{
${ }^{a)}$ Present address: Merck KGaA, Frankfurter Strasse 250, 64293 Darmstadt, Germany.

b)Electronic mail: moritz.riede@iapp.de.
}

(ETL) of $n-\mathrm{C}_{60}$ is used for ohmic contact between ITO and the absorber. The photoactive bulk heterojunction $\left(\mathrm{Ph}_{2^{-}}\right.$ benz-bodipy: $\mathrm{C}_{60}$ ) is embedded between an intrinsic ETL and an additional intrinsic donor layer for better coverage of the infrared region.

$45 \mathrm{~nm}$ of p-doped 9,9-bis[4-(N,N-bis-biphenyl-4-yl-amino) phenyl]-9H-fluorene (BPAPF) is used as hole transport layer. A combination of $1 \mathrm{~nm} \mathrm{Ca}$ with $12-16 \mathrm{~nm} \mathrm{Ag}$ is used as transparent top contact/back electrode. On top of the metal electrode, a 20-150 nm thick capping layer of tris(8-hydroxyquinolinato)-aluminum ( $\left.\mathrm{Alq}_{3}\right)$ is used for increased light in/ outcoupling $^{6}$ out of the solar cell. All organic materials except the dopants had been purified at least twice by vacuum gradient sublimation. Instead of the commonly used tetra-fluoro-tetracyano-quinodimethane $\left(\mathrm{F}_{4}\right.$-TCNQ) or $\mathrm{WO}_{3}$ to p-dope materials with high ionization potentials and acridine orange base (AOB) as n-dopant, NDP9 and NDN1 are used because of a better processability (Novaled AG, Germany). Their doping performance, however, is comparable. ${ }^{9,10}$ Typical solar cell areas are approximately $6.27 \mathrm{~mm}^{2}$, as defined by the overlap of ITO and metal contact, determined with a light microscope. Storage and characterization are under ambient conditions.

The standard $j(V)$ measurements are carried out using simulated AM 1.5 G sun light from a $16 \mathrm{~S}-150$ V.3 sunlight simulator (Solar Light Co., USA). The illumination intensity $I_{0}$ is monitored by a calibrated Si diode; during measurement, $I_{0}=102 \pm 1 \mathrm{~mW} / \mathrm{cm}^{2}$ (corrected for spectral mismatch). External quantum efficiency (EQE) is measured using a monochromator and the lock-in technique (Oriel Xe Arc-Lamp Apex Illuminator combined with Cornerstone 260 $1 / 4 \mathrm{~m}$ monochromator (Newport, USA)) and lock-in amplifier 7265 DSP (Signal Recovery, UK).

The resulting measurements are summarized in Table I. The SMOSC achieve high open circuit voltages $V_{\text {oc }}$ of $0.81 \mathrm{~V}$ if illuminated from the bottom, which is approximately $0.3 \mathrm{~V}$ higher than for the commonly used zinc phthalocyanine and similar to ClAlPc. ${ }^{7}$ The metal layer thickness $t_{\mathrm{Ag}}$ has no influence on $V_{\mathrm{oc}}$, indicating that in all cases, there is excellent electrical contact between organics and electrodes.

Typical short circuit current densities $j_{\mathrm{sc}}$ are 5.3-5.8 mA/ $\mathrm{cm}^{2}$. Thicker metal layers lead to higher photocurrents. The 

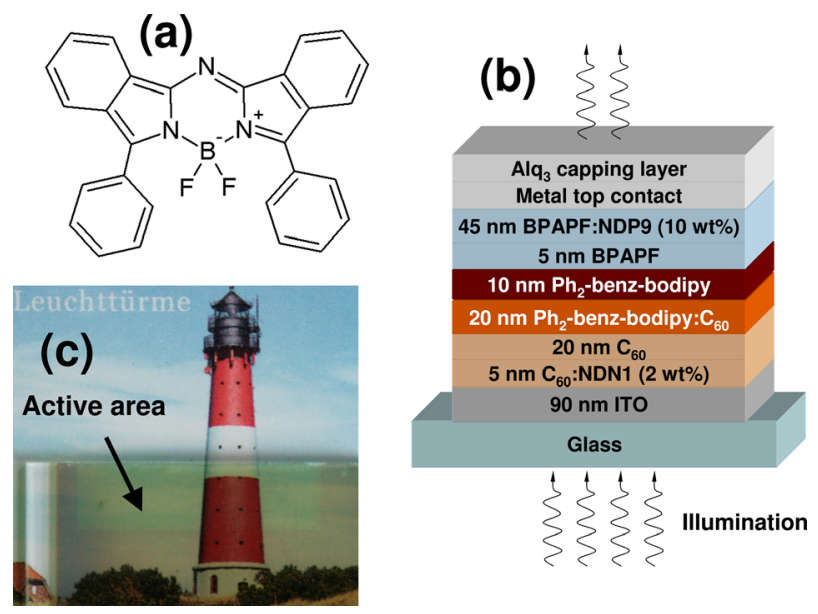

FIG. 1. (Color online) (a) Structure of the absorber material $\mathrm{Ph}_{2}$-benz-bodipy; (b) schematic of the solar cell stack; (c) picture of the lighthouse of Hörnum, photographed with a SMOSC substrate on top. The active area is marked with the black arrow.

difference can be as large as 7\%, comparing $12 \mathrm{~nm}$ and $16 \mathrm{~nm}$ $\mathrm{Ag}$ at $20 \mathrm{~nm}$ capping layer thickness with 5.4 and $5.8 \mathrm{~mA} /$ $\mathrm{cm}^{2}$, respectively. This is explained by the stronger reflection at the metal back electrode if the device is illuminated through the ITO bottom contact: the constructive interference in the absorber layers is enhanced, improving photon absorption. However, at the same time, $T$ through the SMOSC is decreased, such that a compromise must be found for optimized semitransparent applications. The small increase in fill factor (FF) from $51 \%$ to $54 \%$ from $12 \mathrm{~nm} \mathrm{Ag}$ to thicker layers may be related to a slightly better metal morphology, i.e., improved percolation pathways, for thicker metal layers. ${ }^{3}$

The $\mathrm{Alq}_{3}$ capping layer is a second means to controlling interference and hence optical properties and device performance. $^{4,5}$ The data demonstrate that at any given fixed metal thickness, the capping layer thickness $t_{\mathrm{Alq} 3}$ weakly influences the photocurrent by up to $7 \%$ by slightly changing the absorption. As the EQE (Fig. 2 bottom) demonstrates, $t_{\mathrm{Ag}}$ has no strong influence on device properties: $\mathrm{EQE} \approx 40 \%$ at $770 \mathrm{~nm}$ (where $\mathrm{Ph}_{2}$-benz-bodipy absorbs) and $27 \%$ at $350 \mathrm{~nm}$ are achieved for all devices. However, $t_{\text {Alq3 }}$ slightly influences the NIR peak and the range of $400-500 \mathrm{~nm}$, where $\mathrm{C}_{60}$ absorbs, due to thin film optical effects.

TABLE I. Summary of the current-voltage parameters. Top three rows: top illumination and bottom five rows: bottom ilumination. The values of $t_{\mathrm{Ag}}$ and $t_{\mathrm{Alq} 3}$ represent the metal and capping layer thickness, respectively. The average visible transmission $T_{\mathrm{VIS}}$ is calculated in the range from 400$750 \mathrm{~nm}$, the yellow-green $T_{\mathrm{YG}}$ from $495-590 \mathrm{~nm}$.

\begin{tabular}{lrcccccc}
\hline \hline $\begin{array}{c}t_{\mathrm{Ag}} \\
\mathrm{nm}\end{array}$ & $\begin{array}{c}t_{\mathrm{Alq} 3} \\
\mathrm{~nm}\end{array}$ & $\begin{array}{c}V_{\mathrm{oc}} \\
\mathrm{V}\end{array}$ & $\begin{array}{c}j_{\mathrm{sc}} \\
\mathrm{mA} \mathrm{cm}^{-2}\end{array}$ & $\begin{array}{c}\mathrm{FF} \\
\%\end{array}$ & $\begin{array}{c}\eta \\
\%\end{array}$ & $\begin{array}{c}T_{\mathrm{VIS}} \\
\%\end{array}$ & $\begin{array}{c}T_{\mathrm{YG}} \\
\%\end{array}$ \\
\hline 14 & 20 & 0.74 & $1.0 \pm 0.1$ & 59 & $0.4 \pm 0.1$ & 42.8 & 55.7 \\
14 & 75 & 0.75 & $1.2 \pm 0.1$ & 60 & $0.5 \pm 0.1$ & 42.1 & 53.0 \\
14 & 150 & 0.75 & $1.0 \pm 0.1$ & 58 & $0.4 \pm 0.1$ & 31.7 & 39.3 \\
12 & 20 & 0.81 & $5.4 \pm 0.1$ & 51 & $2.2 \pm 0.1$ & 47.9 & 61.8 \\
14 & 20 & 0.81 & $5.7 \pm 0.1$ & 53 & $2.4 \pm 0.1$ & 42.8 & 55.7 \\
16 & 20 & 0.81 & $5.8 \pm 0.1$ & 53 & $2.4 \pm 0.1$ & 36.1 & 46.7 \\
14 & 75 & 0.81 & $5.5 \pm 0.1$ & 53 & $2.3 \pm 0.1$ & 42.1 & 53.0 \\
14 & 150 & 0.81 & $5.3 \pm 0.1$ & 54 & $2.3 \pm 0.1$ & 31.7 & 39.3 \\
\hline \hline
\end{tabular}
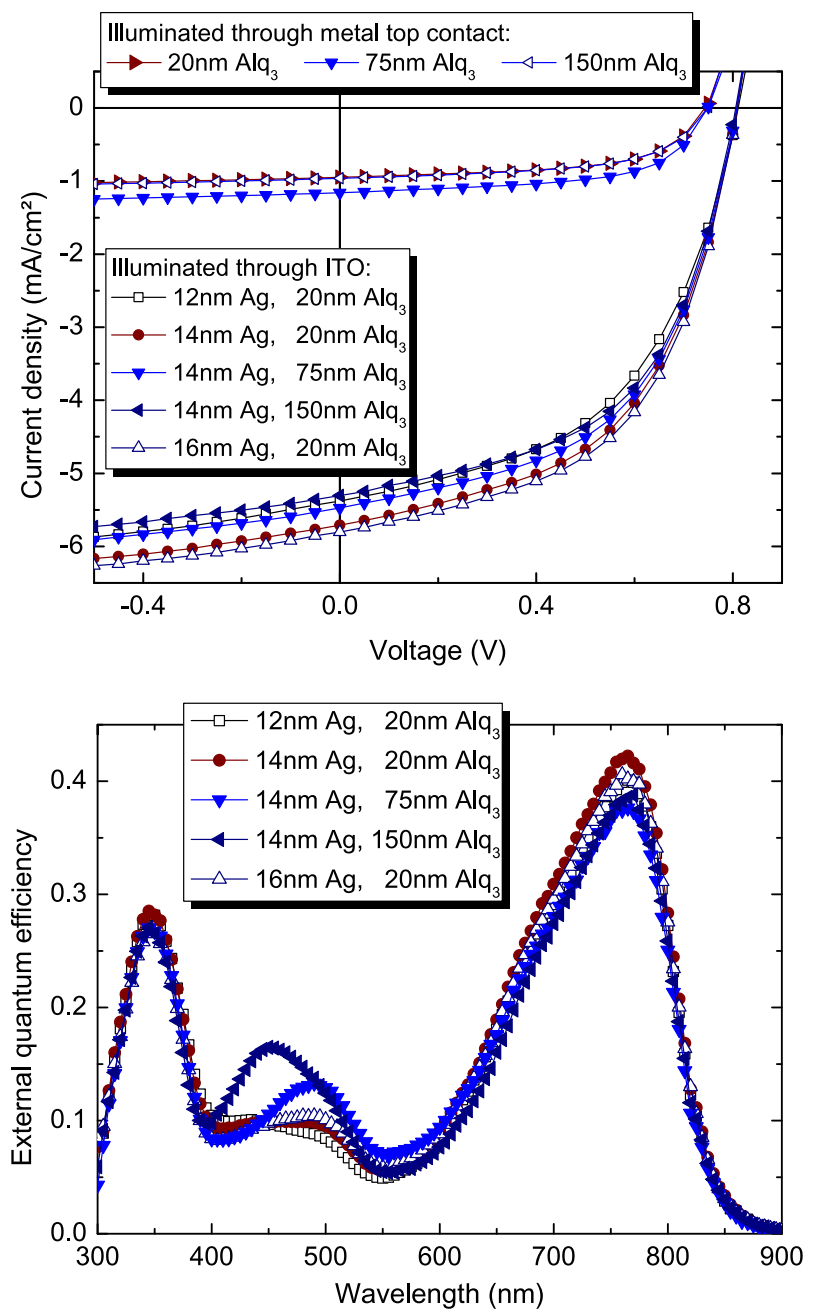

FIG. 2. (Color online) Top: $j(V)$ curves of the SMOSC, illuminated either through the top contact (low current densities) or through the substrate (high current densities). Bottom: $\mathrm{EQE}$, measured through the substrate.

The capping layer can be used to tailor $T$ and reflectance $R$, as shown in Fig. 3. The main influence of higher $t_{\mathrm{Ag}}$ is a lower $T$ in the complete spectrum, with a drop of up to $20 \%$ when comparing $12 \mathrm{~nm}$ and $16 \mathrm{~nm} \mathrm{Ag}$. The capping layer can be used to counter this by increasing $T$ with only minimal losses of $j_{\mathrm{sc}}$ by reducing $R$. This is

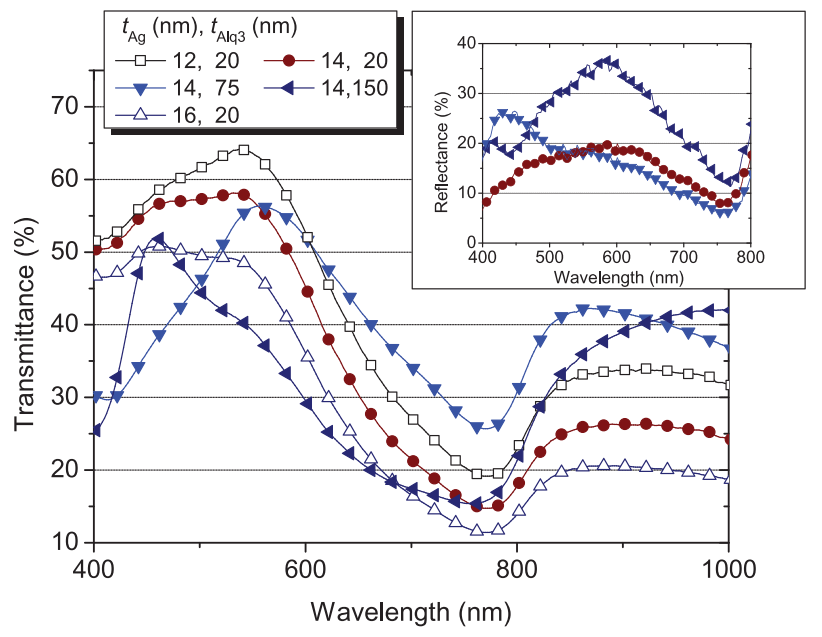

FIG. 3. (Color online) Transmittance and reflectance of the active areas, measured for bottom illumination. 


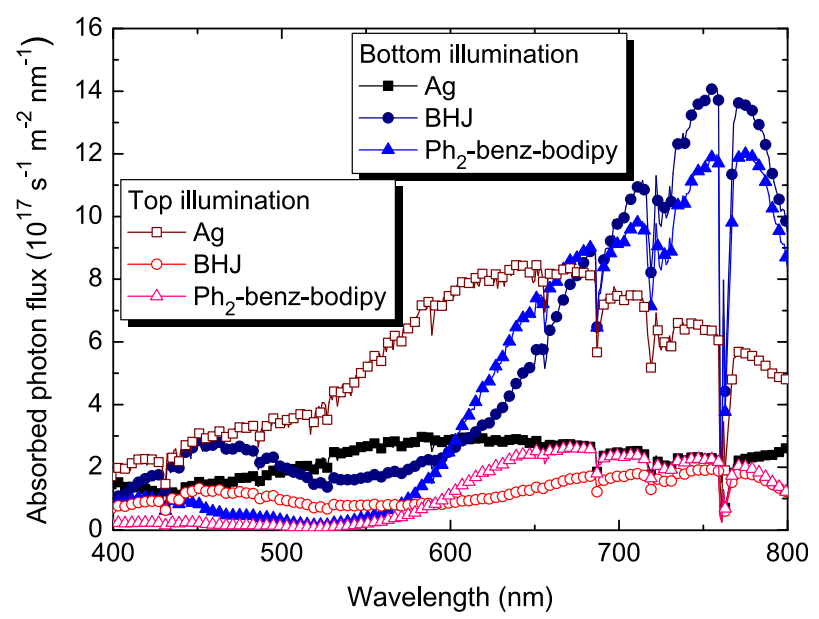

FIG. 4. (Color online) Absorbed photon flux of metal top contact and photoactive layers under simulated AM $1.5 \mathrm{G}$ illumination through the substrate (filled symbols) or through the metal top contact (empty symbols) at $14 \mathrm{~nm}$ $\mathrm{Ag}$ and $75 \mathrm{~nm} \mathrm{Al}_{3}$.

visible in the inset: at $t_{\mathrm{Alq} 3}=75 \mathrm{~nm}, R$ is up to $20 \%$ lower compared to $t_{\mathrm{Alq} 3}=150 \mathrm{~nm}$, corresponding to a comparable increase in $T$.

Furthermore, there are fundamental differences depending on the illumination direction (through the glass substrate and ITO or from the top through $\mathrm{Alq}_{3}$ and metal contact). The transmission is independent of the illumination orientation, but absorption and reflection are strongly influenced. We illustrate this with optical simulations based on the transfer matrix approach ${ }^{11}$ performed with the software osoleMIO. ${ }^{12}$ The absorbed photon fluxes of the bulk heterojunction (BHJ), the intrinsic $\mathrm{Ph}_{2}$-benz-bodipy, and the $\mathrm{Ag}$ layer are shown in Fig. 4. The simulations assume illumination with AM 1.5G light.

The most decisive factor concerning the optical effects, and an important parameter for the photocurrent, is interference at the ultra-thin metal top contact. If light is illuminated through the substrate, a significant part of what is not absorbed in the first pass is reflected at the metal top contact. This results in a second pass through the absorber layers and leads to a high photocurrent due to a microcavity effect. In contrast to that, if the device is illuminated from the top, reflection of the incoming wave is strongly increased; furthermore, the light that is transmitted through the top contact makes only one pass through the absorber layers before being outcoupled though ITO and glass, such that the device performance is significantly lower.

If the device is illuminated through the bottom, there is moderate absorption in the $\mathrm{BHJ}$ in the range where $\mathrm{C}_{60}$ absorbs (400-500 $\mathrm{nm})$. The main feature is the high absorbed photon flux of up to $1.4 \times 10^{18} /\left(\mathrm{s} \mathrm{m}^{2} \mathrm{~nm}\right)$ at $750 \mathrm{~nm}$ due to the significant absorption of $\mathrm{Ph}_{2}$-benz-bodipy. Altogether, the parasitic absorption of the Ag top contact is comparably small, with less than $4 \times 10^{17} /\left(\mathrm{s} \mathrm{m}^{2} \mathrm{~nm}\right)$ at any wavelength.

This is drastically changed when the sample is illuminated from the top (open symbols in Fig. 4). In this case, the Ag exhibits significantly higher absorption than the organic materials, especially in the gap between $\mathrm{C}_{60}$ and $\mathrm{Ph}_{2}$-benzbodipy in the range of $500-600 \mathrm{~nm}$. Much light at higher wavelengths is reflected by the metal; combined with the high parasitic absorption, the optical field density, and photon flux in the absorber layers is strongly weakened, resulting in a drop in $j_{\mathrm{sc}}$ from $>5 \mathrm{~mA} / \mathrm{cm}^{2}$ to $1-1.2 \mathrm{~mA} / \mathrm{cm}^{2}$. The charge carrier density within the device is much smaller with top illumination. This contributes to the significantly lower $V_{\text {oc, }}$ which is $60 \mathrm{mV}$ smaller with top illumination, by reducing the quasi-Fermi level splitting. Despite a higher FF of up to $60 \%$, caused by less recombination due to a smaller charge carrier density, the efficiency drops from $2.2 \%-2.4 \%$ to $0.4 \%-0.5 \%$ if the illumination direction is changed to top illumination.

In conclusion, we present highly efficient semitransparent organic solar cell devices, demonstrating that high transparency (up to $65 \%$ at peak wavelengths) and efficiency $(2.4 \% \pm 0.1 \%)$ are possible with semitransparent metal contacts, provided that suitable NIR absorbers and optimized layers are available.

The current work is supported by the German Federal Ministry of Education and Research (BMBF) in the framework of the InnoProfile (03IP602) project.

\footnotetext{
${ }^{1}$ M. A. Green, K. Emery, Y. Hishikawa, and W. Warta, Prog. Photovoltaics 19, 84 (2011).

${ }^{2}$ A. Henemann, Renewable Energy Focus 9(1), 14 (2008).

${ }^{3}$ J. Meiss, M. K. Riede, and K. Leo, J. Appl. Phys. 105, 063108 (2009).

${ }^{4}$ B. O'Connor, K. H. An, K. P. Pipe, Y. Zhao, and M. Shtein, Appl. Phys. Lett. 89, 233502 (2006).

${ }^{5}$ J. Meiss, N. Allinger, M. K. Riede, and K. Leo, Appl. Phys. Lett. 93, 103311 (2009).

${ }^{6}$ J. Meiss, T. Menke, C. Uhrich, W.-M. Gnehr, S. Sonntag, M. Pfeiffer, K. Leo, and M. K. Riede, Appl. Phys. Lett. 99, 043301 (2011).

${ }^{7}$ R. R. Lunt and V. Bulovic, Appl. Phys. Lett. 98(11), 113305 (2011).

${ }^{8}$ R. Gresser, M. Hummert, H. Hartmann, K. Leo, and M. Riede, Chem. Eur. J. 17, 2939 (2011).

${ }^{9}$ F. Li, M. Pfeiffer, A. G. Werner, K. Harada, K. Leo, N. Hayashi, K. Seki, X. Liu, and X.-D. Dang, J. Appl. Phys. 100, 023716 (2006).

${ }^{10}$ J. Meyer, S. Hamwi, S. Schmale, T. Winkler, H.-H. Johannes, T. Riedl, and W. Kowalsky, J. Mater. Chem. 19, 702 (2009).

${ }^{11}$ E. Centurioni, Appl. Opt. 44(35), 7532 (2005).

${ }^{12}$ developed by Mauro Furno at IAPP.
} 